\title{
Asymptotic analysis of thermoelastic response in functionally graded thin plate subjected to a transient thermal shock
}

\author{
Y. Z. Wang ${ }^{1, *}$, D. Liu ${ }^{1}$, Q. Wang ${ }^{1}$, J. Z. Zhou ${ }^{2}$ \\ ${ }^{1}$ Department of Energy and Power Engineering, Jiangsu University, Zhenjiang 212013, P. R. China \\ ${ }^{2}$ Department of Mechanical Engineering, Jiangsu University, Zhenjiang 212013, P. R. China \\ *Corresponding author: wyz3701320@ujs.edu.cn
}

\begin{abstract}
This paper is concerned with the thermoelastic response of a functionally graded thin plate with an analytical approach. The governing equations are derived in the context of the Lord and Shulman theory (L-S theory), where the material properties of the thin plate are assumed to be graded along the lengthwise direction according to a power law distribution. An asymptotic approach based on the layer method and the Laplace transform technique is presented to deal with these nonlinear governing equations, and then the closed-form solutions of displacement, temperature and stresses, induced by a sudden temperature rise at the boundary, are derived. The propagation of each wave, as well as the distributions of each physical field, are plotted and discussed. The comparison is also conducted to evaluate the effect of characteristic parameter, including the thermal relaxation time and the power law index, on thermoelastic response.
\end{abstract}

Keywords: Generalized thermoelasticity; Functionally graded plate; Asymptotic solutions; Lord-Shulman theory; Thermal shock

\section{Introduction}

In recent years, the functionally graded materials (FGMs), designed to withstand elevated temperature and severe thermal gradient, are widely used in some engineering practices [1]. The analysis of thermoelastic response, especially the prediction of thermal stresses generated in some severe circumstances, is very important to evaluate the lives of FGMs and has received considerate attention. Many researches on the thermoelastic behavior of FGMs have done in the context of the conventional coupled theory of thermoelasticity [2-6]. The conventional coupled theory proposed by Biot [7], however, predicts an infinite speed of heat propagation, which contradicts physical facts and limits the applicability of the results to the 
classical heat conduction. To eliminate this shortcoming, some modified theories, admitting a finite propagation speed of heat signal, are proposed on different perspective [8-13], which are also named as generalized theories of thermoelasticity to distinguish from the conventional coupled theory.

Within these generalized theories, Bagri and Eslami [14] studied the dynamic thermoelastic response of functionally graded annular disk in the assumption that material properties have a power law distribution along the radial direction. Utilizing the same distributions of material properties, Darabseh et al. [15] solved thermal stresses of a thick hollow cylinder composed of FGMs under thermal loading. Ghosh and Kanoria [16] analyzed the distributions of thermoelastic displacement and associate stresses in a functionally graded hollow sphere due to the prescribed temperature, and pointed out that the magnitudes of the displacement and stresses had a strong dependency on the non-homogeneous parameter. Furthermore, the exponential distribution with the spatial position for material properties was also employed to analyze the thermoelastic interaction in FGMs by Mallik and Kanoria [17], Babaei and Chen [18], respectively. Furthermore, Kanoria and Ghosh [19] studied the thermoelastic response of a functionally graded spherically isotropic hollow, where the temperature-dependent properties were considered. Abbas [20] also solved the thermoelastic interaction in a thick-walled FGM cylinder with temperature-dependent properties, and pointed out that temperature dependency of material properties would affect the magnitudes of each physical quantity to some extent.

Since the material properties of FGMs are graded along the spatial position, the material parameters are usually described by a function of the coordinates [21]. Consequently the governing equations become of nonlinear form in general cases, and the theoretical treatment is very difficult. The initial approach is to have a linear treatment to these nonlinear equations by means of some linearization technique with appropriate theoretical approximation, such as the layer method [22]. The merit of this approach is the closed-form solutions can be derived when the governing equations are linearized, which is very convenient to evaluate the lives of FGMs. For the thermoelastic problems involving generalized theories of thermoelasticity, however, the governing equations would become more complicated, the closed-form solutions are almost impossible to obtain even if the equations are linear, which limit the application of 
this linearization approach. Instead, two numerical methods are usually employed in previous investigations, the one is the integral transform with numerical inversion, and the other is the directly solution by means some effective numerical techniques like the finite element method. For the former, the Laplace transform technique is firstly used to have a solution in the transform domain, where some effective approaches, such as the state-space approach [23] and the eigenvalue approach [24] are employed to deal with these complicated equations, and then the numerical inverse technique is introduced to complete the numerical solution in the time domain. Unfortunately the truncation error and the discrete error, generated from the numerical inversion, would reduce the precision of final results [25], especially the jumps locating at each wavefront, which is an important phenomenon induced by the finite propagation speed of heat signal, cannot be captured accurately [26]. For the latter, the complicated integral transform and inverse transform can be avoided even the problem with complicated boundaries can also be solved [27], however, the artificial dissipation, introduced to eliminate the numerical oscillations generated at each wavefront, would smooth the discontinuity of solutions, and leads to too big or too small prediction for magnitudes of peak stresses, which is negative to evaluate the properties of FGMs.

Recently an asymptotic approach [28] is introduced to solve some generalized thermoelastic problems [26, 29]. For this asymptotic approach, the Laplace transform technique and its limit theorem are used to have an analytical solution to these generalized equations, and the closed-form solutions can be obtained when the governing equations are linearized, which is very important to reveal the general phenomenon involving finite propagation speed of heat signal and expands the applicability of linearization technique to generalized thermoelastic problems.

The aim of this paper is to propose an asymptotic approach to solve the thermoelastic response of FGMs generated from severe temperature environments, which is built on the works of Balla [28] and Wang et al. [26, 29], as well as the linear treatment for governing equations by the layer method. The thermoelastic response of a functionally graded thin plate with the boundary subjected to a sudden temperature rise is solved by this asymptotic approach, and the closed-form solutions of the displacement, temperature and stresses are obtained. The explicit expressions of the propagation velocity of each wave and its wavefront 
position are obtained, and the propagation of each wave along the lengthwise direction is also plotted. The distributions of the displacement, temperature and stresses are discussed, and the effect of material properties with a power law distribution as well as the delay effect is also studied.

\section{Formulations of the problem}

The fundamental equations of FGMs derived from the L-S theory [8] in absence of body forces and heat generation are given as

The constitutive equations

$$
\sigma_{i j}=\lambda \gamma_{k k} \delta_{i j}+2 \mu \gamma_{i j}-\beta \theta \delta_{i j}
$$

The equations of motion

$$
\rho \ddot{u}_{i}=\sigma_{i j, j}
$$

The energy balance equation

$$
q_{i, i}=-\rho c_{p} \dot{\theta}-T_{0} \beta \dot{\gamma}_{k k}
$$

The heat conduction equation

$$
q_{i}+\tau_{0} \dot{q}_{i}=-k \theta_{, i} .
$$

The linear strain-displacement relations

$$
\gamma_{i j}=\frac{1}{2}\left(u_{i, j}+u_{j, i}\right)
$$

In the preceding equations, $i, j=1,2,3$ refer to general coordinates, $u_{i}$ are the components of the displacement vector, $q_{i}$ are the components of the heat flux vector, $\sigma_{i j}$ are the components of the stress tensor, $\gamma_{i j}$ are the components of the strain tensor, $\theta=T-T_{0}$ is the increment temperature, $T$ is the absolute temperature, $T_{0}$ is the reference temperature, $\rho$ is the mass density, $k$ is the thermal conductivity, $c_{p}$ is the specific heat at constant strain, $\beta=(3 \lambda+2 \mu) \alpha_{T}$ is the thermal-mechanical coefficient, $\alpha_{T}$ is the coefficient of linear thermal expansion, $\lambda$ and $\mu$ are the Lame's constants, $\tau_{0}$ is the relaxation time constant for L-S theory. Meanwhile, the superscript $\operatorname{dot}(\cdot)$ and the subscript 
comma (, ) denote the derivatives to the time $t$ and coordinates $x_{i}(i=1,2,3)$, respectively.

Since FGMs consist of some constituent materials, mostly are metal and ceramic, the effective properties of FGMs depend on these constituent material properties, and can be expressed as the following function for a ceramic-metal FGM [21]:

$$
P=P_{m} V_{m}+P_{c} V_{c}
$$

where $P$ indicates the effective property of FGM, $P_{m}$ and $P_{c}$ are the properties of metal and ceramic, respectively, and $V_{m}$ and $V_{c}$ are the corresponding volume fractions of metal and ceramic. Meanwhile, the volume fractions satisfy the relation $V_{m}+V_{c}=1$.

We consider an insulated thin plate of thickness $L$ composed of a functionally graded material. The boundaries are traction free and keep the uniform temperature $T_{0}$ initially. For time $t=0$ the surface of boundary $x=0$ is suddenly raised to a constant temperature $T_{1}$.

From the physics of the problem, it is clear that all the physical quantities will depend on $x$ and $t$ only. Thus, the displacement vector has the components:

$$
u_{x}=u(x, t), u_{y}=u_{z}=0 .
$$

Taking account into the linear strain-displacement relations (5), we have

$$
\gamma_{x x}=\frac{\partial u}{\partial x}, \quad \gamma_{y y}=\gamma_{z z}=\gamma_{x y}=\gamma_{x z}=\gamma_{y z}=0 \text {. }
$$

Substituting these strain components into the constitutive equations (1), the non-zero stress components can be derived as

$$
\begin{gathered}
\sigma_{x x}=[\lambda(x)+2 \mu(x)] \frac{\partial u}{\partial x}-\beta(x) \theta, \\
\sigma_{y y}=\sigma_{z z}=\lambda(x) \frac{\partial u}{\partial x}-\beta(x) \theta .
\end{gathered}
$$

Substituting these non-zero stress components into equation of motion (2) results in

$$
\rho(x) \frac{\partial^{2} u}{\partial t^{2}}=[\lambda(x)+2 \mu(x)] \frac{\partial^{2} u}{\partial x^{2}}-\beta(x) \frac{\partial \theta}{\partial x}+\frac{\partial}{\partial x}[\lambda(x)+2 \mu(x)] \frac{\partial u}{\partial x}-\frac{\partial \beta(x)}{\partial x} \theta .
$$

Combining Eq. (3) with Eq. (4) to derive the temperature equation as

$$
k(x) \frac{\partial^{2} \theta}{\partial x^{2}}+\frac{\partial k(x)}{\partial x} \frac{\partial \theta}{\partial x}=\rho(x) c_{p}(x)\left(\tau_{0} \frac{\partial^{2} \theta}{\partial t^{2}}+\frac{\partial \theta}{\partial t}\right)+T_{0} \beta(x)\left(\tau_{0} \frac{\partial^{3} u}{\partial t^{2} \partial x}+\frac{\partial^{2} u}{\partial t \partial x}\right)
$$

The initial and boundary conditions take the forms: 


$$
\begin{gathered}
t=0: \quad u=0, \frac{\partial u}{\partial t}=0, T=T_{0}, \frac{\partial T}{\partial t}=0, \\
x=0: \quad T=T_{0}+\left(T_{1}-T_{0}\right) H(t), \sigma_{x x}=0, \\
x=L: T=T_{0}, \quad \sigma_{x x}=0,
\end{gathered}
$$

where $H(t)$ is the Heaviside unit function.

For convenience of following solutions, some non-dimensional variables are introduced:

$$
x^{*}=a v_{e} x, \quad L^{*}=a v_{e} L, t^{*}=a v_{e}^{2} t, \tau_{0}^{*}=a v_{e}^{2} \tau_{0}, \theta^{*}=\frac{\theta}{T_{0}}, u^{*}=a v_{e} \frac{\lambda_{m}+2 \mu_{m}}{\beta_{m} T_{0}} u, \quad \sigma_{i i}^{*}=\frac{\sigma_{i i}}{\beta_{m} T_{0}}
$$

Substituting these non-dimensional variables into above equations (9)-(12) and dropping the asterisks for convenience, we have

$$
\begin{gathered}
\sigma_{x x}=f(\lambda+2 \mu) \frac{\partial u}{\partial x}-f(\beta) \theta \\
\sigma_{y y}=\sigma_{z z}=k_{\lambda} f(\lambda+2 \mu) \frac{\partial u}{\partial x}-f(\beta) \theta \\
f(\rho) \frac{\partial^{2} u}{\partial t^{2}}=f(\lambda+2 \mu) \frac{\partial^{2} u}{\partial x^{2}}-f(\beta) \frac{\partial \theta}{\partial x}+\frac{\partial f(\lambda+2 \mu)}{\partial x} \frac{\partial u}{\partial x}-\frac{\partial f(\beta)}{\partial x} \theta \\
f(k) \frac{\partial^{2} \theta}{\partial x^{2}}+\frac{\partial f(k)}{\partial x} \frac{\partial \theta}{\partial x}=f\left(\rho c_{p}\right)\left(\tau_{0} \frac{\partial^{2} \theta}{\partial t^{2}}+\frac{\partial \theta}{\partial t}\right)+f(\beta) \vartheta\left(\tau_{0} \frac{\partial^{3} u}{\partial t^{2} \partial x}+\frac{\partial^{2} u}{\partial t \partial x}\right)
\end{gathered}
$$

where $a=\frac{\rho_{m} c_{p_{m}}}{k_{m}}$ is the thermal viscosity constant, $v_{e}=\sqrt{\frac{\lambda_{m}+2 \mu_{m}}{\rho_{m}}}$ is the standard speed of thermoelastic wave, $\vartheta=\frac{T_{0} \beta_{m}^{2}}{\rho_{m} c_{p_{m}}\left(\lambda_{m}+2 \mu_{m}\right)}$ is the thermoelastic coupling constants, $k_{\lambda}=\frac{\lambda_{m}}{\lambda_{m}+2 \mu_{m}}$ is the non-dimensional constants, and $f(P)$ is the non-dimensional function of each material parameter with respect to coordinate $x$.

As discussed by Eq. (7), the material properties of FGM are the space-dependent function, and this functional relationship may be assumed to have a power law form [2-6, 21]. For this reason, the metal volume fraction $V_{m}$ for a functionally grade thin plate with the thickness $L$ may be introduced as

$$
V_{m}=(x / L)^{z_{0}}
$$


where $z_{0}$ is power law index that constitutes the metal constituent richness in functionally graded thin plate.

Substituting above equation in Eq. (7), we can have

$$
f(P)=P_{m}\left[\left(\frac{x}{L}\right)^{z}\left(1-\frac{P_{c}}{P_{m}}\right)+\frac{P_{c}}{P_{m}}\right] .
$$

The non-dimensional forms of the initial and boundary conditions take the forms:

$$
\begin{gathered}
t=0: u=0, \frac{\partial u}{\partial t}=0, \quad \theta=0, \frac{\partial \theta}{\partial t}=0, \\
x=0: \theta=\theta_{1} H(t), \sigma_{x x}=0, \\
x=L: \theta=0, \quad \sigma_{x x}=0,
\end{gathered}
$$

where $\theta_{1}=\left(T_{1}-T_{0}\right) / T_{0}$.

\section{Asymptotic solutions of the problem}

\subsection{Analytical model of composite laminated plate}

Obviously the governing equations (18) and (19) become nonlinear once inserting the material function (21), which are very difficult to have a theoretical treatment. Here a layer method [22] is introduced to deal with these nonlinear equations, where a composite laminated plate with a large number of layers, shown in Fig.1, is introduced to replace the initial functional graded thin plate. The material parameters $P^{i}=P(i \Delta x)$ of this composite laminated plate, including the Lame's constants $\lambda\left(x_{i}\right)$ and $\mu\left(x_{i}\right)$, the thermal-mechanical coefficient $\beta\left(x_{i}\right)$, the mass density $\rho\left(x_{i}\right)$, the thermal conductivity $k\left(x_{i}\right)$ and the specific heat $c_{p}\left(x_{i}\right)$, are graded change with different layer but are constant in the same layer. 


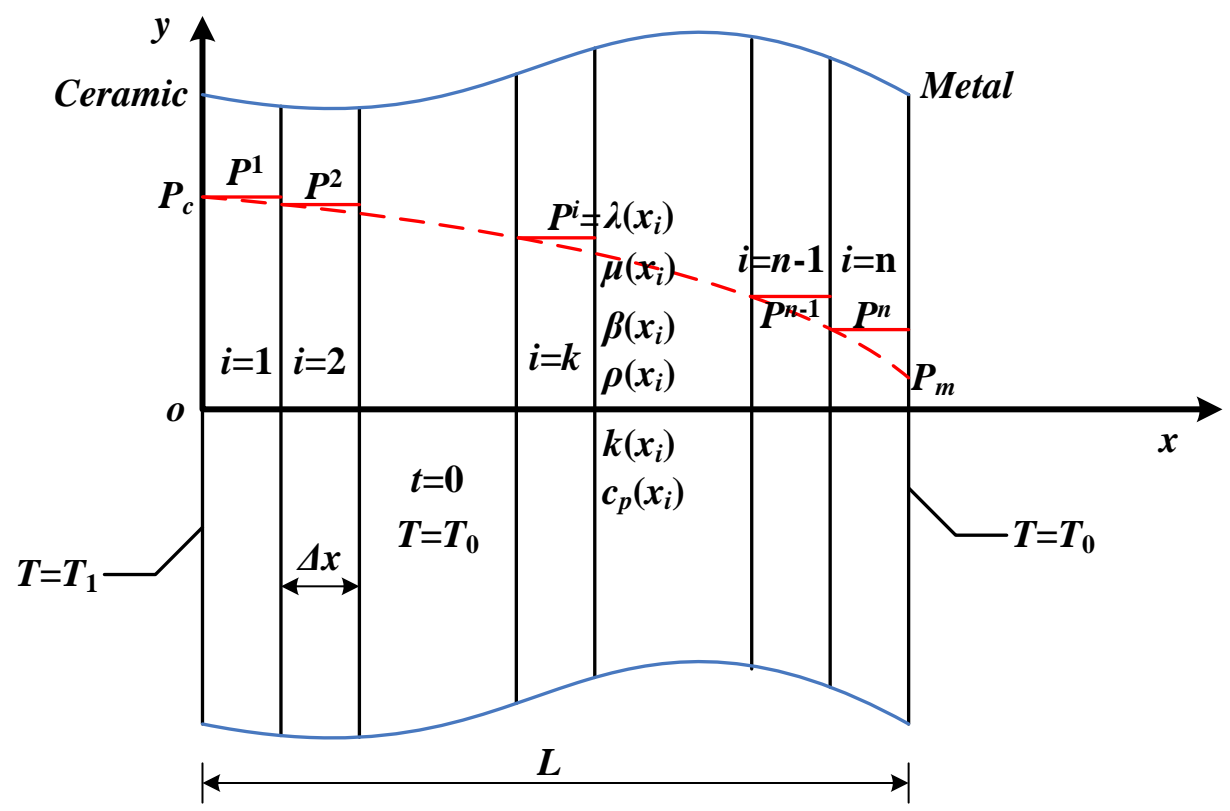

Fig.1. Analytical model of the $n$-layer with distinct isotropic material properties in each layer

Substituting these constant material parameters $\lambda\left(x_{i}\right), \mu\left(x_{i}\right), \beta\left(x_{i}\right), \rho\left(x_{i}\right), k\left(x_{i}\right)$ and $c_{p}\left(x_{i}\right)$ into governing equations (16)-(19), the linear equations for $i$ th layer can be derived as

$$
\begin{gathered}
\sigma_{x x}^{i}=f^{i}(\lambda+2 \mu) \frac{\partial u^{i}}{\partial x}-f^{i}(\beta) \theta^{i}, \\
\sigma_{y y}^{i}=\sigma_{z z}^{i}=k_{\lambda} f^{i}(\lambda+2 \mu) \frac{\partial u^{i}}{\partial x}-f^{i}(\beta) \theta^{i}, \\
f^{i}(\rho) \frac{\partial^{2} u^{i}}{\partial t^{2}}=f^{i}(\lambda+2 \mu) \frac{\partial^{2} u^{i}}{\partial x^{2}}-f^{i}(\beta) \frac{\partial \theta^{i}}{\partial x}, \\
f^{i}(k) \frac{\partial^{2} \theta}{\partial x^{2}}=\bar{f}^{i}\left(\rho c_{p}\right)\left(\tau_{0} \frac{\partial^{2} \theta^{i}}{\partial t^{2}}+\frac{\partial \theta^{i}}{\partial t}\right)+f^{i}(\beta) \vartheta\left(\tau_{0} \frac{\partial^{3} u^{i}}{\partial t^{2} \partial x}+\frac{\partial^{2} u^{i}}{\partial t \partial x}\right),
\end{gathered}
$$

where $u^{i}, \theta^{i}$ and $\sigma_{j j}^{i}(j=1,2,3)$ are the non-dimensional displacement, non-dimensional temperature and non-dimensional stress components of $i$ th layer, respectively, $f^{i}(P)=\frac{P_{c}}{P_{m}}+\left(1-\frac{P_{c}}{P_{m}}\right)\left(\frac{i}{n}\right)^{z_{0}}$, and $\bar{f}^{i}(P)=\frac{P_{c}}{P_{m}}+\left(1-\frac{P_{c}}{P_{m}}\right)\left(\frac{i}{n}\right)^{2 z_{0}}$.

The non-dimensional initial and boundary conditions with layer forms can be rewritten as 


$$
\begin{gathered}
t=0: u^{i}=0, \frac{\partial u^{i}}{\partial t}=0, \theta^{i}=0, \frac{\partial \theta^{i}}{\partial t}=0(i=1,2, \cdots n), \\
x=0: \underline{\theta^{1}=\theta_{1} H(t)}, \underline{\sigma_{x x}^{1}=0} \\
x=L: \underline{\theta^{n}=0,}, \underline{\sigma_{x x}^{n}=0}
\end{gathered}
$$

The interface between adjacent layers is assumed to be perfect conductively and bonded for the functional behavior of FGMs, and then we have

$$
\underline{u^{i}\left(x_{i}, t\right)=u^{i+1}\left(x_{i}, t\right)}, \underline{\theta^{i}\left(x_{i}, t\right)=\theta^{i+1}\left(x_{i}, t\right)}, \underline{\frac{\partial \theta^{i}}{\partial x}=\frac{k^{i+1}}{k^{i}} \frac{\partial \theta^{i+1}}{\partial x}}, \underline{\sigma_{r r}^{i}\left(x_{i}, t\right)=\sigma_{r r}^{i+1}\left(x_{i}, t\right)}
$$

$(i=1,2 \cdots n-1)$

\subsection{Analytical solutions in the transform domain}

Applying the Laplace transform for the both sides of Eqs. (25)-(28), which is defined as

$$
L\{t\}=\bar{f}(s)=\int_{0}^{\infty} e^{-s t} f(t) \mathrm{d} t
$$

Combining with the homogeneous initial condition (29), we have

$$
\begin{gathered}
\bar{\sigma}_{x x}^{i}=f^{i}(\lambda+2 \mu) \frac{\mathrm{d} \bar{u}^{i}}{\mathrm{~d} x}-f^{i}(\beta) \bar{\theta}^{i}, \\
\bar{\sigma}_{y y}^{i}=\bar{\sigma}_{z z}^{i}=k_{\lambda} f^{i}(\lambda+2 \mu) \frac{\mathrm{d} \bar{u}^{i}}{\mathrm{~d} x}-f^{i}(\beta) \bar{\theta}^{i}, \\
f^{i}(\rho) s^{2} \bar{u}^{i}=f^{i}(\lambda+2 \mu) \frac{\mathrm{d}^{2} \bar{u}^{i}}{\mathrm{~d} x^{2}}-f^{i}(\beta) \frac{\mathrm{d} \bar{\theta}^{i}}{\mathrm{~d} x} \\
f^{i}(k) \frac{\mathrm{d}^{2} \bar{\theta}^{i}}{\mathrm{~d} x^{2}}=\bar{f}^{i}\left(\rho c_{p}\right)\left(\tau_{0} s^{2}+s\right) \bar{\theta}^{i}+f^{i}(\beta) \vartheta\left(\tau_{0} s^{2}+s\right) \frac{\mathrm{d} \bar{u}^{i}}{\mathrm{~d} x} .
\end{gathered}
$$

Eliminating terms $\bar{u}^{i}$ and $\bar{\theta}^{i}$ separately by combining Eq. (35) and Eq. (36) results in

$$
\left\{\frac{\mathrm{d}^{4}}{\mathrm{~d} x^{4}}-\left[\begin{array}{l}
\frac{f^{i}(\rho)}{f^{i}(\lambda+2 \mu)} s^{2}+\frac{\bar{f}^{i}\left(\rho c_{p}\right)}{f^{i}(k)}\left(\tau_{0} s^{2}+s\right)+ \\
\frac{\left[f^{i}(\beta)\right]^{2}}{f^{i}(\lambda+2 \mu) f^{i}(k)} \vartheta\left(\tau_{0} s^{2}+s\right)
\end{array}\right] \frac{\mathrm{d}^{2}}{\mathrm{~d} x^{2}}+\frac{f^{i}(\rho) \bar{f}^{i}\left(\rho c_{p}\right)}{f^{i}(\lambda+2 \mu) f^{i}(k)} s^{2}\left(\tau_{0} s^{2}+s\right)\right\}\left(\begin{array}{l}
\bar{u}^{i} \\
\bar{\theta}^{i}
\end{array}\right)=0 .
$$


The general solution of Eq. (37) can be expressed as

$$
\left(\begin{array}{c}
\bar{u}^{i} \\
\bar{\theta}^{i}
\end{array}\right)=\left(\begin{array}{llll}
A_{1}^{i}(s) & B_{1}^{i}(s) & C_{1}^{i}(s) & D_{1}^{i}(s) \\
A_{2}^{i}(s) & B_{2}^{i}(s) & C_{2}^{i}(s) & D_{2}^{i}(s)
\end{array}\right)\left(\begin{array}{c}
\exp \left(R_{1}^{i} x\right) \\
\exp \left(R_{2}^{i} x\right) \\
\exp \left[R_{1}^{i}(L-x)\right] \\
\exp \left[R_{2}(L-x)\right]
\end{array}\right),
$$

where $A_{j}^{i}(s), B_{j}^{i}(s), C_{j}^{i}(s)$ and $D_{j}^{i}(s)(j=1,2)$ are coefficients depending on parameter $S$ and are determined by the given boundary conditions. $R_{1}^{i}$ and $R_{2}^{i}$ are the negative roots of the following characteristic equation:

$$
R^{4}-\left[\begin{array}{l}
\frac{f^{i}(\rho)}{f^{i}(\lambda+2 \mu)} s^{2}+\frac{\bar{f}^{i}\left(\rho c_{p}\right)}{f^{i}(k)}\left(\tau_{0} s^{2}+s\right)+ \\
\frac{f^{i}(\beta)^{2}}{f^{i}(\lambda+2 \mu) f^{i}(k)} \vartheta\left(\tau_{0} s^{2}+s\right)
\end{array}\right] R^{2}+\frac{f^{i}(\rho) \bar{f}^{i}\left(\rho c_{p}\right)}{f^{i}(\lambda+2 \mu) f^{i}(k)} s^{2}\left(\tau_{0} s^{2}+s\right)=0 .
$$

Substituting the solution (37) into Eq. (35) or Eq. (36) results in

$$
\begin{aligned}
& A_{2}^{i}(s)=\frac{f^{i}(\lambda+2 \mu)\left(R_{1}^{i}\right)^{2}-f^{i}(\rho) s^{2}}{f^{i}(\beta) R_{1}^{i}} A_{1}^{i}(s) \quad, \quad B_{2}^{i}(s)=\frac{f^{i}(\lambda+2 \mu)\left(R_{2}^{i}\right)^{2}-f^{i}(\rho) s^{2}}{f^{i}(\beta) R_{2}^{i}} B_{1}^{i}(s), \\
& C_{2}^{i}(s)=-\frac{f^{i}(\lambda+2 \mu)\left(R_{1}^{i}\right)^{2}-f^{i}(\rho) s^{2}}{f^{i}(\beta) R_{1}^{i}} C_{1}^{i}(s), \quad D_{2}^{i}(s)=-\frac{f^{i}(\lambda+2 \mu)\left(R_{2}^{i}\right)^{2}-f^{i}(\rho) s^{2}}{f^{i}(\beta) R_{2}^{i}} D_{1}^{i}(s) .
\end{aligned}
$$

Substituting these expressions (40) into general solution (38) and combining with stress component (33), we have

$$
\begin{aligned}
\bar{\sigma}_{x x}^{i}=\frac{f^{i}(\rho) s^{2}}{R_{1}^{i}}\left\{A_{1}^{i}(s) \exp \left(R_{1}^{i} x\right)-C_{1}^{i}(s) \exp \left[R_{1}^{i}(L-x)\right]\right\}+ \\
\frac{f^{i}(\rho) s^{2}}{R_{2}^{i}}\left\{B_{1}^{i}(s) \exp \left(R_{2}^{i} x\right)-D_{1}^{i}(s) \exp \left[R_{2}^{i}(L-x)\right]\right\} .
\end{aligned}
$$

Applying the Laplace transform to the boundary conditions (30) and (31) and combining with the solutions (38) and (41), we have

$$
\begin{gathered}
A_{1}^{i}(s)=\frac{\theta_{1} R_{1}^{i} f^{i}(\beta) / f^{i}(\lambda+2 \mu)}{\left[\left(R_{1}^{i}\right)^{2}-\left(R_{2}^{i}\right)^{2}\right]\left[1-\exp \left(2 L R_{1}^{i}\right)\right] s}, \quad B_{1}^{i}(s)=-\frac{\theta_{1} R_{2}^{i} f^{i}(\beta) / f^{i}(\lambda+2 \mu)}{\left[\left(R_{1}^{i}\right)^{2}-\left(R_{2}^{i}\right)^{2}\right]\left[1-\exp \left(2 L R_{2}^{i}\right)\right] s}, \\
C_{1}^{i}(s)=A_{1}^{i}(s) \exp \left(L R_{1}^{i}\right), \quad D_{1}^{i}(s)=B_{1}^{i}(s) \exp \left(L R_{1}^{i}\right)
\end{gathered}
$$

Utilizing these expressions, the general solutions of the displacement, temperature and 
stresses in the transform domain can be obtained as

$$
\left(\begin{array}{c}
\bar{u}^{i} \\
\bar{\theta}^{i} \\
\bar{\sigma}_{x x}^{i} \\
\bar{\sigma}_{y y(z z)}^{i}
\end{array}\right)=\frac{f^{i}(\beta)}{f^{i}(\lambda+2 \mu)}\left(\begin{array}{cccc}
F_{1}\left(R_{1}^{i}\right) & -F_{1}\left(R_{2}^{i}\right) & F_{1}\left(R_{1}^{i}\right) & -F_{1}\left(R_{2}^{i}\right) \\
F_{2}\left(R_{1}^{i}\right) & -F_{2}\left(R_{2}^{i}\right) & -F_{2}\left(R_{1}^{i}\right) & F_{2}\left(R_{2}^{i}\right) \\
F_{3}\left(R_{1}^{i}\right) & -F_{3}\left(R_{2}^{i}\right) & -F_{3}\left(R_{1}^{i}\right) & F_{3}\left(R_{2}^{i}\right) \\
F_{4}\left(R_{1}^{i}\right) & -F_{4}\left(R_{2}^{i}\right) & -F_{4}\left(R_{1}^{i}\right) & F_{4}\left(R_{2}^{i}\right)
\end{array}\right)\left(\begin{array}{c}
\frac{\theta_{1} \exp \left(R_{1}^{i} x\right)}{1-\exp \left(2 L R_{1}^{i}\right)} \\
\frac{\theta_{1} \exp \left(R_{2}^{i} x\right)}{1-\exp \left(2 L R_{2}^{i}\right)} \\
\frac{\theta_{1} \exp \left[R_{1}^{i}(2 L-x)\right]}{1-\exp \left(2 L R_{1}^{i}\right)} \\
\frac{\theta_{1} \exp \left[R_{2}^{i}(2 L-x)\right]}{1-\exp \left(2 L R_{2}^{i}\right)}
\end{array}\right),
$$

where $\quad F_{1}(R)=\frac{R}{s\left[\left(R_{1}^{i}\right)^{2}-\left(R_{2}^{i}\right)^{2}\right]} \quad, \quad F_{2}(R)=\frac{f^{i}(\lambda+2 \mu) R^{2}-f^{i}(\rho) s^{2}}{s\left[\left(R_{1}^{i}\right)^{2}-\left(R_{2}^{i}\right)^{2}\right] f^{i}(\beta)}$

$F_{3}(R)=\frac{f^{i}(\rho) R s}{\left(R_{1}^{i}\right)^{2}-\left(R_{2}^{i}\right)^{2}}, \quad F_{4}(R)=\frac{k_{v} f^{i}(\lambda+2 \mu) R^{2}+f^{i}(\rho) s^{2}}{s\left[\left(R_{1}^{i}\right)^{2}-\left(R_{2}^{i}\right)^{2}\right]}, k_{v}=k_{\lambda}-1$.

\subsection{Asymptotic solutions in the time domain}

It is very difficult to construct exact solutions by means of directly inverse Laplace transform for such complicated expressions of roots $R_{1}^{i}$ and $R_{2}^{i}$ defined by characteristic equation (39). Thus, some approximations of roots $R_{1}^{i}$ and $R_{2}^{i}$ are introduced by means of limit theorem of Laplace transform, where transient property of thermal shock problem is considered to obtain the following approximations [26, 28 and 29]:

$$
R_{1,2}^{i}=-k_{1,2}^{i} s-m_{1,2}^{i},
$$

where $\quad k_{1,2}^{i}=\left[\frac{1}{2}\left(\frac{f^{i}(\rho)}{f^{i}(\lambda+2 \mu)}+\frac{\bar{f}^{i}\left(\rho c_{p}\right)}{f^{i}(k)} \tau_{0}+\frac{f^{i}(\beta)^{2}}{f^{i}(\lambda+2 \mu) f^{i}(k)} \vartheta \tau_{0} \pm \sqrt{a^{i}}\right)\right]^{1 / 2}$

$$
\begin{aligned}
& m_{1,2}^{i}=\frac{\left[\frac{\bar{f}^{i}\left(\rho c_{p}\right)}{f^{i}(k)}+\frac{f^{i}(\beta)^{2}}{f^{i}(\lambda+2 \mu) f^{i}(k)} \vartheta\right] \pm \frac{b^{i}}{\sqrt{a^{i}}}}{4 k_{1,2}^{i}} \\
& a^{i}=\left[\frac{f^{i}(\rho)}{f^{i}(\lambda+2 \mu)}+\frac{\bar{f}^{i}\left(\rho c_{p}\right)}{f^{i}(k)} \tau_{0}+\frac{f^{i}(\beta)^{2}}{f^{i}(\lambda+2 \mu) f^{i}(k)} \vartheta \tau_{0}\right]^{2}-\frac{4 f^{i}(\rho) \bar{f}^{i}\left(\rho c_{p}\right)}{f^{i}(\lambda+2 \mu) f^{i}(k)} \tau_{0}
\end{aligned}
$$




$$
b^{i}=\left[\frac{\bar{f}^{i}\left(\rho c_{p}\right)}{f^{i}(k)}+\frac{f^{i}(\beta)^{2}}{f^{i}(\lambda+2 \mu) f^{i}(k)} \vartheta\right]^{2} \tau_{0}+\frac{f^{i}(\rho)}{f^{i}(\lambda+2 \mu) f^{i}(k)}\left[\frac{f^{i}(\beta)^{2}}{f^{i}(\lambda+2 \mu)} \vartheta-\bar{f}^{i}\left(\rho c_{p}\right)\right] .
$$

Substituting these approximations (44) into general solutions (43), the forms are convenient to inverse Laplace transform can be obtained. Using the standard results of Laplace transform technique, the asymptotic solutions of $\bar{u}^{i}, \bar{T}^{i}$ and $\bar{\sigma}_{j j}^{i}(j=1,2,3)$ in the time domain can be derived as

$$
\begin{aligned}
& u^{i}=-\left[u_{k_{1}^{i}}(x)+\sum_{j=1}^{n n} \frac{1}{(j-1) !} u_{k_{1}^{i}}(2 j L+x)\right]+\left[u_{k_{2}^{i}}(x)+\sum_{j=1}^{n n} \frac{1}{(j-1) !} u_{k_{2}^{i}}(2 j L+x)\right]- \\
& {\left[u_{k_{1}^{i}}(2 L-x)+\sum_{j=1}^{n n} \frac{1}{(j-1) !} u_{k_{1}^{i}}(2 j L+2 L-x)\right]+\left[u_{k_{2}^{j}}(2 L-x)+\sum_{j=1}^{n n} \frac{1}{(j-1) !} u_{k_{2}^{j_{2}}}(2 j L+2 L-x)\right]^{\prime}} \\
& \theta^{i}=\left[\theta_{k_{1}^{i}}(x)+\sum_{j=1}^{n n} \frac{1}{(j-1) !} \theta_{k_{1}^{i}}(2 i L+x)\right]-\left[\theta_{k_{2}^{i}}(x)+\sum_{j=1}^{n n} \frac{1}{(j-1) !} \theta_{k_{2}^{i}}(2 j L+x)\right]- \\
& {\left[\theta_{k_{1}^{i}}(2 L-x)+\sum_{j=1}^{n n} \frac{1}{(j-1) !} \theta_{k_{1}^{i}}(2 j L+2 L-x)\right]+\left[\theta_{k_{2}^{j}}(2 L-x)+\sum_{j=1}^{n n} \frac{1}{(j-1) !} \theta_{k_{2}^{j_{2}}}(2 j L+2 L-x)\right]} \\
& \sigma_{x x}^{i}=\left[\sigma_{k_{1}^{\prime}}(x)+\sum_{j=1}^{n n} \frac{1}{(j-1) !} \sigma_{k_{1}^{i}}(2 j L+x)\right]-\left[\sigma_{k_{2}^{i}}(x)+\sum_{j=1}^{n n} \frac{1}{(j-1) !} \sigma_{k_{2}^{\prime}}(2 j L+x)\right]- \\
& {\left[\sigma_{k_{1}^{i}}(2 L-x)+\sum_{j=1}^{n n} \frac{1}{(j-1) !} \sigma_{k_{1}^{i}}(2 j L+2 L-x)\right]+\left[\sigma_{k_{2}^{i}}(2 L-x)+\sum_{j=1}^{n n} \frac{1}{(j-1) !} \sigma_{k_{2}^{i}}(2 j L+2 L-x)\right]^{\prime}} \\
& \sigma_{y y}^{i}=\sigma_{z z}^{i}=\left[\bar{\sigma}_{k_{1}^{i}}(x)+\sum_{j=1}^{n n} \frac{1}{(j-1) !} \bar{k}_{k_{1}^{i}}(2 j L+x)\right]-\left[\bar{\sigma}_{k_{2}^{i}}(x)+\sum_{j=1}^{n n} \frac{1}{(j-1) !} \bar{\sigma}_{k_{2}^{i}}(2 j L+x)\right]- \\
& {\left[\bar{\sigma}_{k_{1}^{\prime}}(2 L-x)+\sum_{j=1}^{n n} \frac{1}{(j-1) !} \bar{\sigma}_{k_{1}^{j_{1}}}(2 j L+2 L-x)\right]+\left[\bar{\sigma}_{k_{2}^{\prime}}(2 L-x)+\sum_{j=1}^{n n} \frac{1}{(j-1) !} \bar{\sigma}_{k_{2}^{\prime}}(2 j L+2 L-x)\right]^{\prime}}
\end{aligned}
$$

where

$$
u_{k_{1}^{i}}(x)=\frac{\theta_{1} f^{i}(\beta)}{\sqrt{a^{i}} f^{i}(\lambda+2 \mu)} \exp \left(-m_{1}^{i} x\right) k_{1}^{i}\left(t-k_{1}^{i} x\right) H\left(t-k_{1}^{i} x\right)
$$$$
u_{k_{2}^{i}}(x)=\frac{\theta_{1} f^{i}(\beta)}{\sqrt{a^{i}} f^{i}(\lambda+2 \mu)} \exp \left(-m_{2}^{i} x\right) k_{2}^{i}\left(t-k_{2}^{i} x\right) H\left(t-k_{2}^{i} x\right)
$$ 


$$
\begin{aligned}
& \theta_{k_{1}^{i}}(x)=\frac{\theta_{1}}{\sqrt{a^{i}}} \exp \left(-m_{1}^{i} x\right)\left[k_{3}^{i}+\left(m_{3}^{i}-\frac{b^{i}}{a^{i}} k_{3}^{i}\right)\left(t-k_{1}^{i} x\right)\right] H\left(t-k_{1}^{i} x\right) \\
& \theta_{k_{2}^{i}}(x)=\frac{\theta_{1}}{\sqrt{a^{i}}} \exp \left(-m_{2}^{i} x\right)\left[k_{4}^{i}+\left(m_{4}^{i}-\frac{b^{i}}{a^{i}} k_{4}^{i}\right)\left(t-k_{2}^{i} x\right)\right] H\left(t-k_{2}^{i} x\right) \\
& \sigma_{k_{1}^{i}}(x)=\frac{\theta_{1} f^{i}(\rho) f^{i}(\beta)}{\sqrt{a^{i}} f^{i}(\lambda+2 \mu)} \exp \left(-m_{1}^{i} x\right)\left[1-\frac{b^{i}}{a^{i}}\left(t-k_{1}^{i} x\right)\right] H\left(t-k_{1}^{i} x\right) \\
& \sigma_{k_{2}^{i}}(x)=\frac{\theta_{1} f^{i}(\rho) f^{i}(\beta)}{\sqrt{a^{i}} f^{i}(\lambda+2 \mu)} \exp \left(-m_{2}^{i} x\right)\left[1-\frac{b^{i}}{a^{i}}\left(t-k_{2}^{i} x\right)\right] H\left(t-k_{2}^{i} x\right) \\
& \bar{\sigma}_{k_{1}^{i}}(x)=\frac{\theta_{1}}{\sqrt{a^{i}}} \exp \left(-m_{1}^{i} x\right)\left\{k_{5}^{i}+\left[k_{v} m_{3}^{i}-\frac{b^{i}}{a^{i}} k_{5}^{i}\right]\left(t-k_{1}^{i} x\right)\right\} H\left(t-k_{1}^{i} x\right) \\
& \bar{\sigma}_{k_{2}^{i}}(x)=\frac{\theta_{1}}{\sqrt{a^{i}}} \exp \left(-m_{2}^{i} x\right)\left\{k_{6}^{i}+\left[k_{v} m_{4}^{i}-\frac{b^{i}}{a^{i}} k_{6}^{i}\right]\left(t-k_{2}^{i} x\right)\right\} H\left(t-k_{2}^{i} x\right) \\
& k_{3,4}^{i}=\frac{1}{2}\left(-\frac{f^{i}(\rho)}{f^{i}(\lambda+2 \mu)}+\frac{\bar{f}^{i}\left(\rho c_{p}\right)}{f^{i}(k)} \tau_{0}+\frac{f^{i}(\beta)^{2}}{f^{i}(\lambda+2 \mu) f^{i}(k)} \vartheta \tau_{0} \pm \sqrt{a^{i}}\right) \\
& m_{3,4}^{i}=\frac{\left[\frac{\bar{f}^{i}\left(\rho c_{p}\right)}{f^{i}(k)}+\frac{f^{i}(\beta)^{2}}{f^{i}(\lambda+2 \mu) f^{i}(k)} \vartheta\right] \pm \frac{b^{i}}{\sqrt{a^{i}}}}{2} \bar{f}_{5,6}^{i}=\frac{k_{v} f^{i}(\beta)}{2}\left(-\frac{f^{i}(\rho)}{f^{i}(\lambda+2 \mu)}+\frac{f^{i}(\beta)^{2}}{f^{i}(k)} \tau_{0}+\frac{f^{i}(\lambda+2 \mu) f^{i}(k)}{\left.f_{0} \pm \sqrt{a^{i}}\right)+\frac{f^{i}(\beta)}{f^{i}(\lambda+2 \mu)} .} .\right.
\end{aligned}
$$

\section{Numerical solutions and discussion}

It is necessary to validate these closed-form solutions before going to the coming analysis. Since the previous investigations mainly focus on the thermoelastic response taking place at the half-space or axisymmetric body, the same case is difficult to present. Considering the solutions of half-space problem, which contains an unbounded boundary, should be a special case of present closed-form solutions due to the superposition of boundary conditions, these closed-form solutions would reduce to the following forms if we ignore the reflection of waves and have a constant material properties:

$$
u=-\frac{\theta_{1} \exp \left(-m_{1} x\right)}{\sqrt{a}} k_{1}\left(t-k_{1} x\right) H\left(t-k_{1} x\right)+\frac{\theta_{1} \exp \left(-m_{2} x\right)}{\sqrt{a}} k_{2}\left(t-k_{2} x\right) H\left(t-k_{2} x\right)
$$




$$
\begin{aligned}
& \theta=\frac{\theta_{1} \exp \left(-m_{1} x\right)}{\sqrt{a}}\left[k_{3}+\left(m_{3}-k_{3} \frac{b}{a}\right)\left(t-k_{1} x\right)\right] H\left(t-k_{1} x\right)- \\
& \frac{\theta_{1} \exp \left(-m_{2} x\right)}{\sqrt{a}}\left[k_{4}+\left(m_{4}-k_{4} \frac{b}{a}\right)\left(t-k_{2} x\right)\right] H\left(t-k_{2} x\right) \\
& \sigma_{x x}=\frac{\theta_{1} \exp \left(-m_{1} x\right)}{\sqrt{a}}\left[1-\frac{b}{a}\left(t-k_{1} x\right)\right] H\left(t-k_{1} x\right)-\frac{\theta_{1} \exp \left(-m_{2} x\right)}{\sqrt{a}}\left[1-\frac{b}{a}\left(t-k_{2} x\right)\right] H\left(t-k_{2} x\right) \\
& \sigma_{y y}=\sigma_{z z}=\frac{\theta_{1} \exp \left(-m_{1} x\right)}{\sqrt{a}}\left[k_{5}+\left(k_{w} m_{3}-\frac{b}{a} k_{5}\right)\left(t-k_{1} x\right)\right] H\left(t-k_{1} x\right)- \\
& \frac{\theta_{1} \exp \left(-m_{2} x\right)}{\sqrt{a}}\left[k_{6}+\left(k_{w} m_{4}-\frac{b}{a} k_{6}\right)\left(t-k_{2} x\right)\right] H\left(t-k_{2} x\right)
\end{aligned}
$$

These are the same of the solutions of half-space problems proposed by the previous investigations [26, 28], which indicates the correctness of these closed-form solutions.

Now for the illustration of these asymptotic layer solutions obtained in preceding Sections, the functional graded thin plate composed of Zirconia $\left(\mathrm{ZrO}_{2}\right)$ and Titanium (Ti-6Al-4V) is considered in this paper. The thermo-mechanical properties of these materials at room temperature are shown in Table 1[15]. Furthermore, the other constants for calculations are taken as:

$$
\tau_{0}=0.5, \underline{\vartheta=0.0032}, k_{\lambda}=0.473, \theta_{1}=1, L=2, T_{0}=300 \mathrm{~K}, \underline{n=400 .} .
$$

Table 1 Material properties of Titanium and Zirconia at room temperature ${ }^{[15]}$

\begin{tabular}{ccccccc}
\hline material & $k, \mathrm{~W} /(\mathrm{mK})$ & $E, \mathrm{GPa}$ & $\alpha_{T}, 1 / \mathrm{K}$ & $v$ & $\rho, \mathrm{kg} / \mathrm{m}^{3}$ & $\mathrm{c}_{\mathrm{p}}, \mathrm{J} /(\mathrm{kgK})$ \\
\hline $\mathrm{Ti}-6 \mathrm{Al}-4 \mathrm{~V}$ & 18.1 & 66.2 & $10.3 \times 10^{-6}$ & 0.321 & $4.42 \times 10^{3}$ & 808.3 \\
$\mathrm{ZrO}_{2}$ & 2.036 & 117.0 & $7.11 \times 10^{-6}$ & 0.333 & $5.6 \times 10^{3}$ & 615.6 \\
\hline
\end{tabular}

\subsection{Analysis of wave propagation}

Although these asymptotic solutions (45)-(48) seem to be complicated, which consist of four parts for each solution and each part includes two terms, we still observe that two waves, named as thermoelastic wave and thermal wave, respectively, would generate from the boundary by the properties of Heaviside unit function, where the first two parts describe the propagation of two waves, and the other two parts indicate the reflections of two waves when they arrive at the other thermal insulating boundary. Furthermore, the second term of each 
part, such as $\sum_{j=1}^{n n} \frac{1}{(j-1) !} u_{k_{1}^{i}}(2 j L+x)$ for the first part of solution (45), indicates the superposition of each wave, and the value of parameter $n n$ depends on the duration of thermal shock.

Due to the properties of Heaviside unit function, the velocities of two waves reaching $i$ th layer can be derived as

$$
v_{1,2}^{i}=1 / k_{1,2}^{i} \text {. }
$$

Utilizing these expressions, the continuous velocities of two waves in the whole plate and corresponding positions of wavefront can also be obtained by inserting the layer thickness $\Delta x \rightarrow 0$ into the Eq. (49):

$$
v_{1,2}=1 / k_{1,2}, \quad \xi_{1,2}^{\mathrm{iw}}=t / k_{1,2}-j L(j=2,4, \cdots 2 n), \quad \xi_{1,2}^{\mathrm{rw}}=(j+1) L-t / k_{1,2}(j=1,3, \cdots 2 n-1) \text {. }
$$

where $j=\left\lfloor t / k_{1,2}\right\rfloor$, parameters $k_{1,2}$ can be obtained from $k_{1,2}^{i}$ by replacing the function $f^{i}(P)$ into $f(P)$, and superscripts "iw" and "rw" indicate the incident wave and reflection wave, respectively.

Due to the expressions of $k_{1,2}$, we can observe that both velocities are dependent on the thermal relaxation time $\tau_{0}$, the thermoelastic coupling coefficient $\vartheta$ and the power law index $z_{0}$. If ignoring the delay effect, that is $\tau_{0} \rightarrow 0$, the governing equation (12) would reduce to the classical heat conduction equation, and we have $v_{1} \rightarrow \sqrt{f(\lambda+2 \mu) / f(\rho)}$ and $v_{2} \rightarrow \infty$. This means $v_{2}$ indicates the propagation velocity of thermal wave, which is infinite for the Fourier heat conduction, and $v_{1}$ indicates the propagation velocity of thermoelastic wave.

Figures 2-3 display the velocity distributions of two waves at all positions for different values of thermal relaxation time constant $\tau_{0}$ and power law index $z_{0}$, respectively. With the increase of $\tau_{0}$, which means the delay effect is enhancing, both velocities at all positions are decreased for given value of power law index $\left(z_{0}=3\right)$. Meanwhile, this decrease of velocity for thermoelastic wave is increased but is an opposite effect for thermal wave, which 
means the propagation of thermoelastic wave is more sensitive to delay effect than thermal wave. Furthermore, we also observe both velocities at all positions have a non-monotonic distribution for small value of $\tau_{0}\left(\tau_{0} \leq 0.75\right)$, but this non-monotonic distribution would tend to be monotonic with the increase of $\tau_{0}$. Combining with the spatial dependence of material parameters for FGMs, we can conclude that the velocities of two waves are also space-dependent, but this spatial dependence is weakened with the enhancement of delay effect. This can also be found from the velocity distributions of two waves for different values of power law index $z_{0}$ plotted in Fig.3.

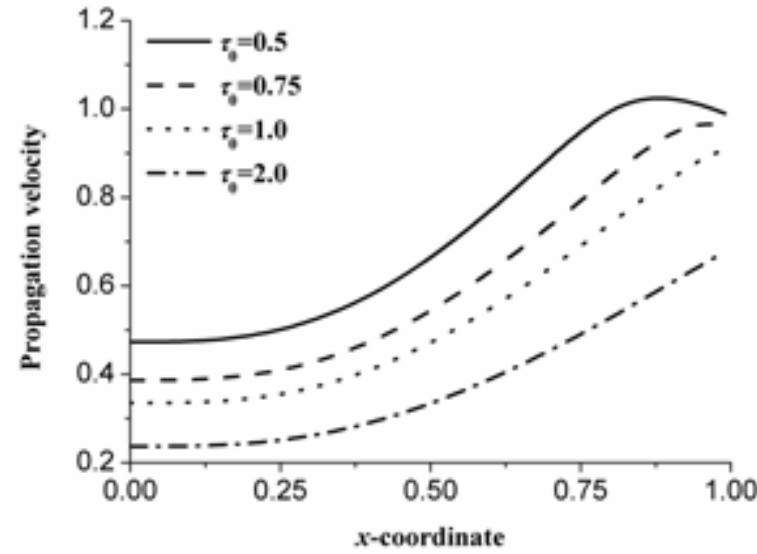

(a) thermoelastic wave

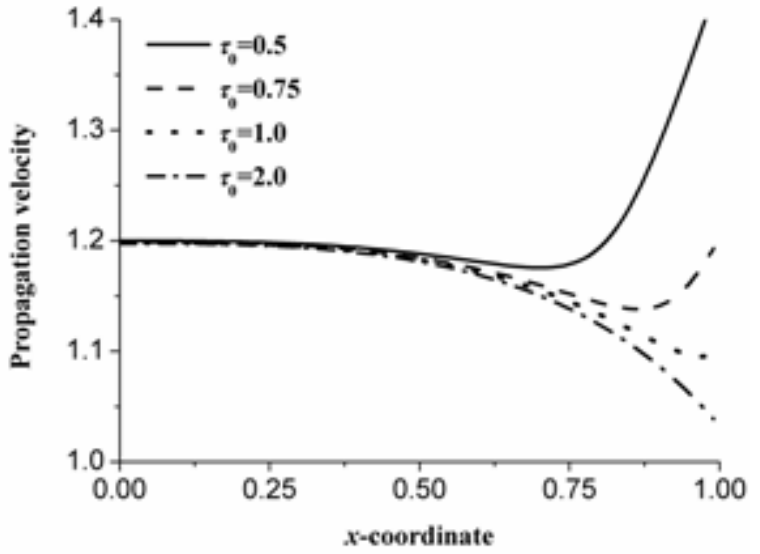

(b) thermal wave

Fig.2. The distributions of propagation velocities $v_{1,2}$ versus $x$ with different thermal relaxation time $\tau_{0}$

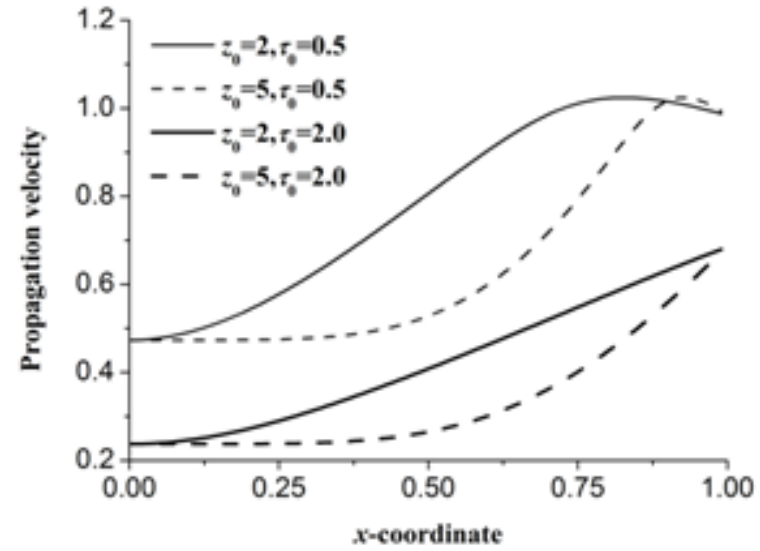

(a) thermoelastic wave

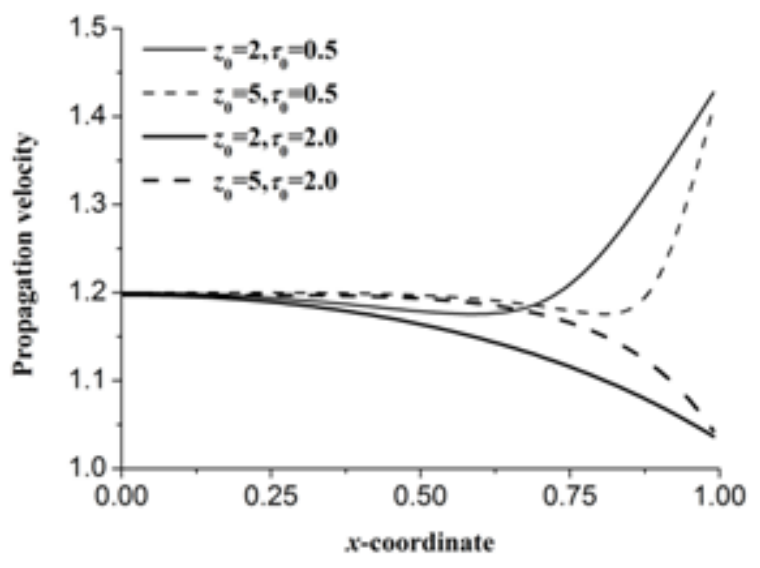

(b) thermal wave

Fig.3. The distributions of propagation velocities $v_{1,2}$ versus $x$ with different power law index $z$

\subsection{Analysis of thermoelastic response}


Utilizing above asymptotic layer solutions (45)-(48), the distributions of displacement $u^{i}$, temperature $\theta^{i}$ and each stress component $\sigma_{j j}^{i}$ for $i$ th layer can be obtained, and these asymptotic layer solutions of $u^{i}, \theta^{i}$ and $\sigma_{j j}^{i}$ can approach the real solutions of $u, \theta$ and $\sigma_{j j}$ for $\Delta x \rightarrow 0$.

Figures 4-6 display the non-dimensional distributions of displacement $u$, temperature $\theta$ and stress component $\sigma_{j j}$ at all positions for different time $t$ and given parameters $\left(z_{0}=3, \tau_{0}=0.5\right)$, where $t=0.1,0.25,0.5$ and 0.8 corresponds to the cases of propagations and reflections of two waves, respectively. Due to the expressions (50), we have $v_{1}<v_{2}$ for the given calculation constants, that is thermal wave propagates faster than thermoelastic wave. The time reaching the other boundary for two waves can be derived as $t_{1}=1.017$ and $t_{2}=0.695$. Obviously, the wavefront of faster wave has not reached the other side for $t \leq 0.5$, the general phenomena involving finite propagation speed of heat signal can be clearly observed from these distributions, which are all of $u, \theta$ and $\sigma_{j j}$ vanish at all positions beyond the faster wavefront. The displacement has a continuous distribution but the temperature and stresses are discontinuous, and two jumps would generate in each wavefront, although the second jump for temperature distribution is very small and can't be observed clearly. This is an important phenomenon and can't be captured by numerical methods employed by previous investigations. Furthermore, the peak stress would generate in each wavefront, whose magnitudes are very important to evaluate the life of FGMs. Fortunately the magnitudes of these peak stress can also be obtained by substituting wavefronts $\xi_{1,2}^{\text {rw }}$ into solutions (47) and (48), which can be expressed as

$$
\begin{aligned}
& {\left[\sigma_{x x}\right]_{\xi_{1}^{\mathrm{ww}}}=\frac{\theta_{1} f(\rho) f(\beta)}{\sqrt{a} f(\lambda+2 \mu)}\left\{\exp \left(-m_{1} \xi_{1}^{\mathrm{rw}}\right)-\exp \left(-m_{2} \xi_{1}^{\mathrm{rw}}\right)\left[1-\frac{b}{a} k_{2}\left(\xi_{2}^{\mathrm{rw}}-\xi_{1}^{\mathrm{rw}}\right)\right]\right\}} \\
& {\left[\sigma_{x x}\right]_{\xi_{2}^{\mathrm{ww}}}=-\frac{\theta_{1} f(\rho) f(\beta)}{\sqrt{a} f(\lambda+2 \mu)} \exp \left(-m_{2} \xi_{2}^{\mathrm{rw}}\right)} \\
& {\left[\sigma_{y y(z z)}\right]_{\xi_{1}^{\mathrm{rw}}}=\frac{\theta_{1}}{\sqrt{a}}\left\{k_{5} \exp \left(-m_{1} \xi_{1}^{\mathrm{rw}}\right)-\exp \left(-m_{2} \xi_{1}^{\mathrm{rw}}\right)\left[k_{6}+\left(k_{v} m_{4}-\frac{b}{a} k_{6}\right) k_{2}\left(\xi_{2}^{\mathrm{rw}}-\xi_{1}^{\mathrm{rw}}\right)\right]\right\}}
\end{aligned}
$$


$\left[\sigma_{y y(z z)}\right]_{\xi_{1}^{\mathrm{rw}}}=\frac{\theta_{1}}{\sqrt{a}} k_{6} \exp \left(-m_{2} \xi_{2}^{\mathrm{rw}}\right), \quad\left(t<k_{2} L, \Delta x \rightarrow 0\right)$.

As the time goes on, the magnitudes of displacement at boundary and the peak stresses are increased by the absorption of heat. For $t=0.8$, the faster wave has reached the other side and began to reflect, meanwhile, the displacement, temperature and stresses near the reflection boundary have a significant change by the superposition of waves. Furthermore, it is noted that the values of temperature and stress component at two boundaries are $\theta=1$, $\sigma_{x x}=0$ for $x=0$, and $\theta=0, \sigma_{x x}=0$ for $x=L$, which is consistent with the boundary conditions (23) and (24) and validate the correctness of present solutions.

Furthermore, the effect of the layer numbers used to calculation is also obtained in the Table 2 for given parameters $\left(z_{0}=3, \tau_{0}=0.5, t=0.5\right)$. The values of each physical quantity at the location of each wavefront, including the non-displacement, non-temperature and non-stress component, observed from Table 2 are stabilized with the increase of the layer numbers. More numbers over $n=400$ does not change the values very considerably, and the layer numbers $n=400$ is therefore accepted as the reasonable size for calculations.

Table 2 Numerical convergence on the numbers of layers

\begin{tabular}{ccccc}
\hline Layer number & {$[u]_{\xi_{1}^{\mathrm{rw}}}$} & {$[\theta]_{\xi_{1}^{\mathrm{rw}}}$} & {$\left[\sigma_{x x}\right]_{\xi_{1}^{\mathrm{rw}}}$} & {$\left[\sigma_{x x}\right]_{\xi_{2}^{\mathrm{fw}}}$} \\
\hline$n=100$ & 0.05177 & 0.61523 & -0.08382 & -0.88445 \\
$n=200$ & 0.05129 & 0.60928 & -0.08337 & -0.85870 \\
$n=400$ & 0.05121 & 0.60877 & -0.08315 & -0.84626 \\
$n=800$ & 0.05109 & 0.60851 & -0.08304 & -0.84014 \\
\hline
\end{tabular}

Figures 7-9 demonstrates the variations of each distribution for different thermal relaxation time $\tau_{0}=(0.5,1.0,2.0)$ and given parameters $\left(t=0.5, z_{0}=3\right)$. The thermal relaxation time is a very important characteristic parameter for generalized thermoelastic problems, which indicates the delay effect between heat flux and temperature gradient and admits the finite speed of heat signal. With the increase of $\tau_{0}$, the propagations of two waves would be difficult by the enhancing of the delay effect, which leads to the decrease of response regions. Meanwhile the magnitudes of the displacement at boundary and the peak 
stresses are also decreased, respectively. It is noted that the position of thermoelastic wavefront has a larger change than that of thermal wavefront for the variation of $\tau_{0}$ from the stress distribution plotted in Fig. 9, which means the propagation of thermoelastic wave is more dependent on the delay effect. This phenomenon can also be observed in Fig.2, where the velocity variation is increased for thermoelastic wave but is decreased for thermal wave with the values of $\tau_{0}$ increasing.

The effect of power law index $z_{0}$ on displacement, temperature and stresses at response regions for the given parameter $\left(t=0.5, \tau_{0}=0.5\right)$ is shown in Figures 10-12, where the power law index $z_{0}=2,3$ and 5 . It is seen that the variations of displacement and stresses at all position of the region $0 \leq x \leq \xi_{1}^{\text {iw }}$ are not significant wit the increase of $z_{0}$, but the magnitude of displacement is increased and the stresses are decreased for the region $\xi_{1}^{\mathrm{iw}} \leq x \leq \xi_{2}^{\mathrm{iw}}$. The temperature and peak stresses are decreased at whole response region with the increase of $z_{0}$, which means the thermal shock effect imposed on the boundary can be reduced when the FGMs is used as a thermal barrier. In addition, the similar effect on propagations of each wave to thermal relaxation time is also observed from the stress component distribution plotted in Fig.12, except from the variation of wavefront positions for two waves are different with $z_{0}$ increasing, the wavefront position of thermoelastic wave is increased but is decreased for thermal wave.

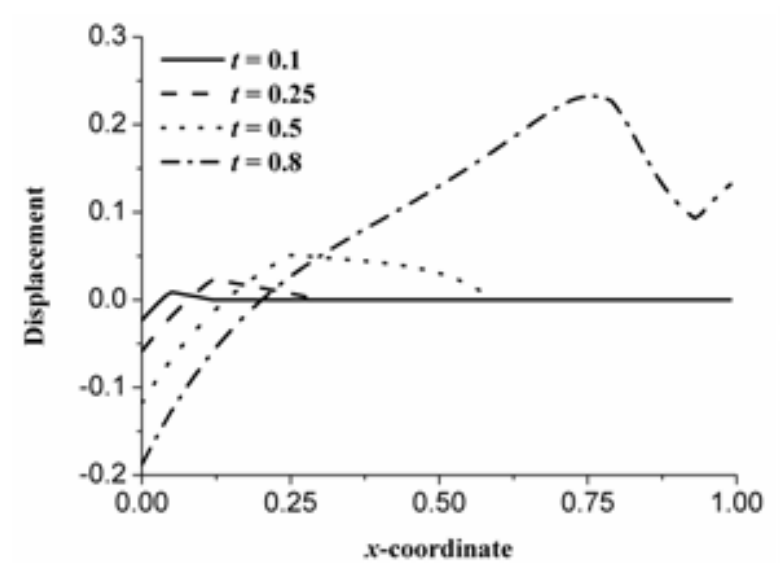

Fig.4. Distribution of displacement $u$ versus $x$ at different time $t$

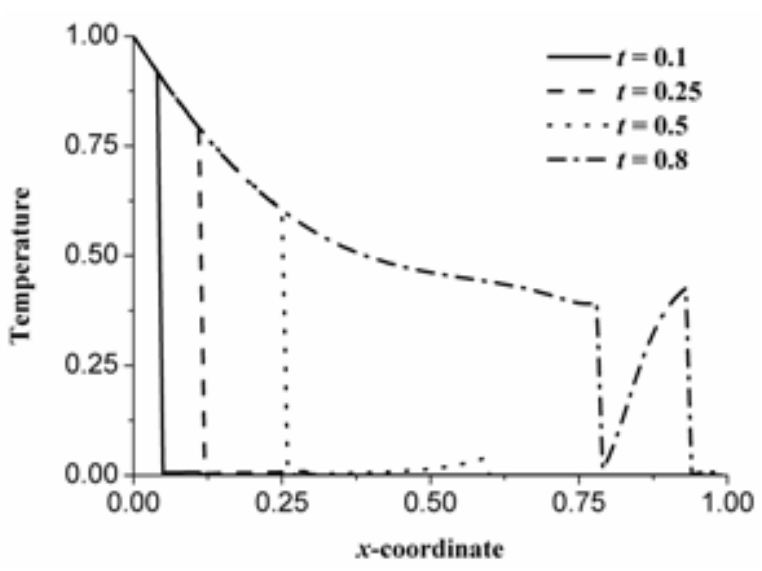

Fig.5. Distribution of temperature $\theta$ versus $x$ at different time $t$ 


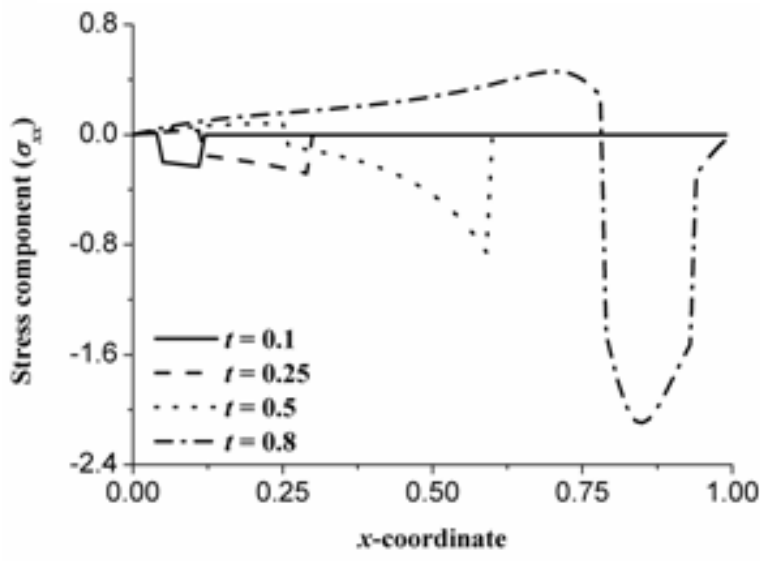

(a)

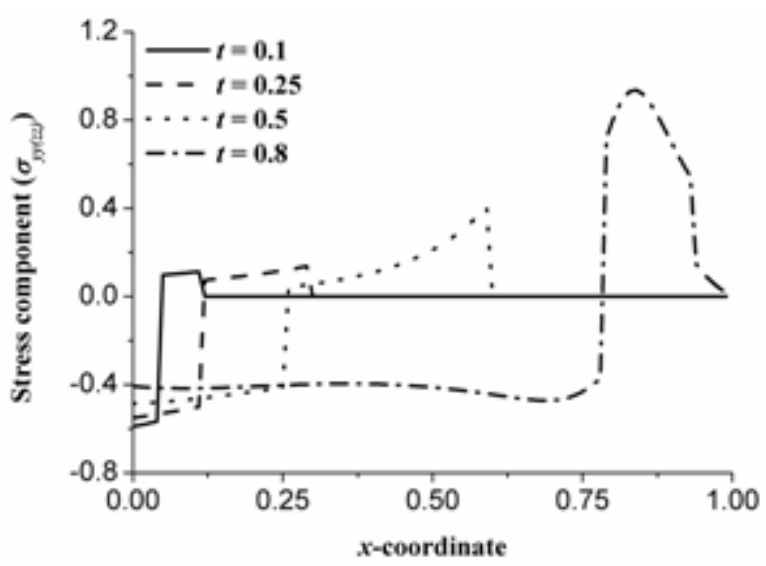

(b)

Fig.6. Distribution of stress components $\sigma_{i i}(i=1,2,3)$ versus $x$ at different time $t$

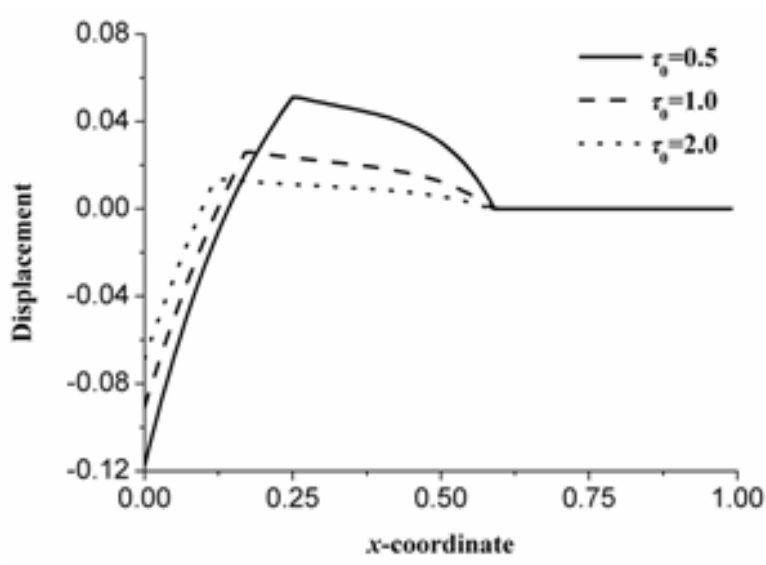

Fig.7. Distribution of displacement $u$ versus $x$ with different thermal relaxation time $\tau_{0}$

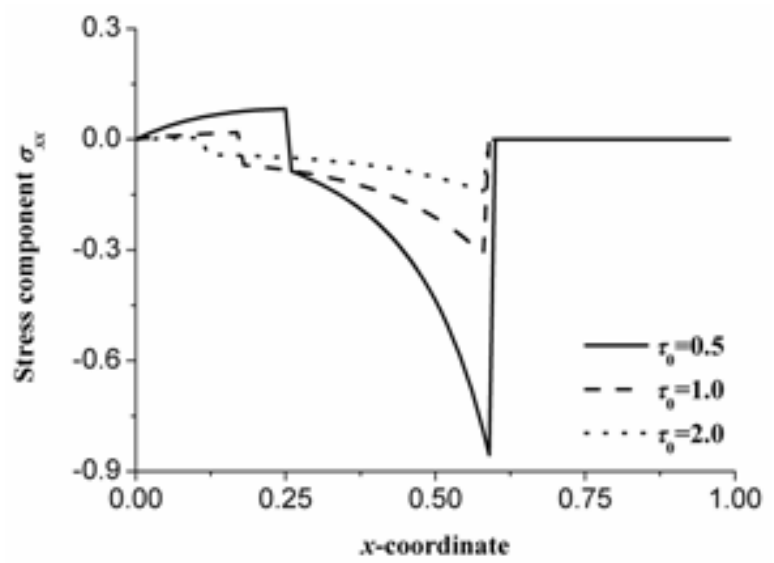

Fig.9. Distribution of stress component $\sigma_{x x}$ versus $x$ with different thermal relaxation time $\tau_{0}$

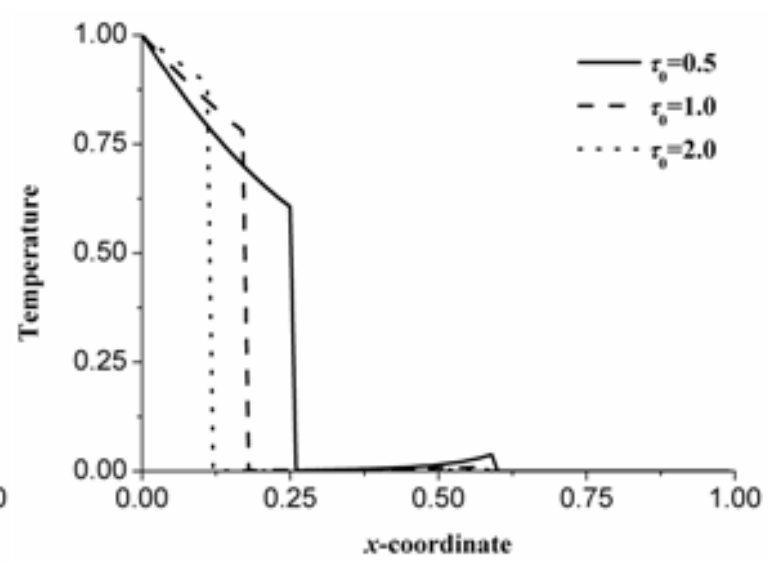

Fig.8. Distribution of temperature $\theta$ versus $x$ with different thermal relaxation time $\tau_{0}$

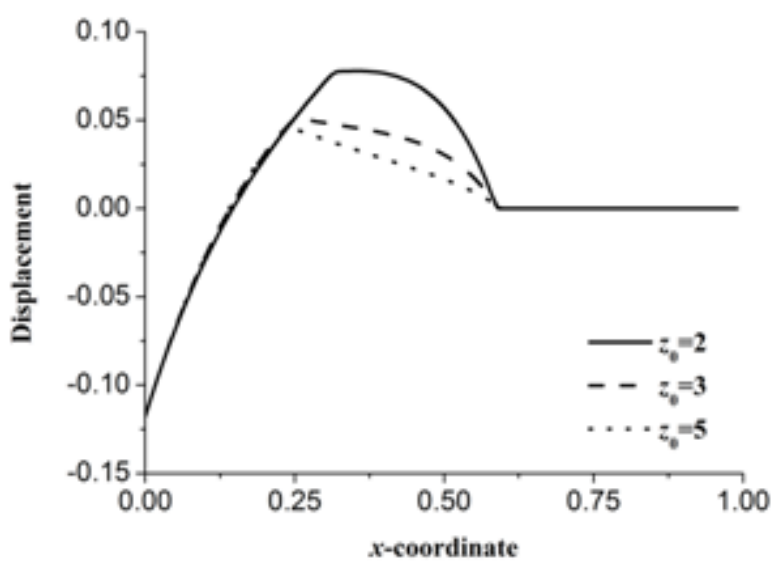

Fig.10. Distribution of displacement $u$ versus $x$ with different power law index $z$ 


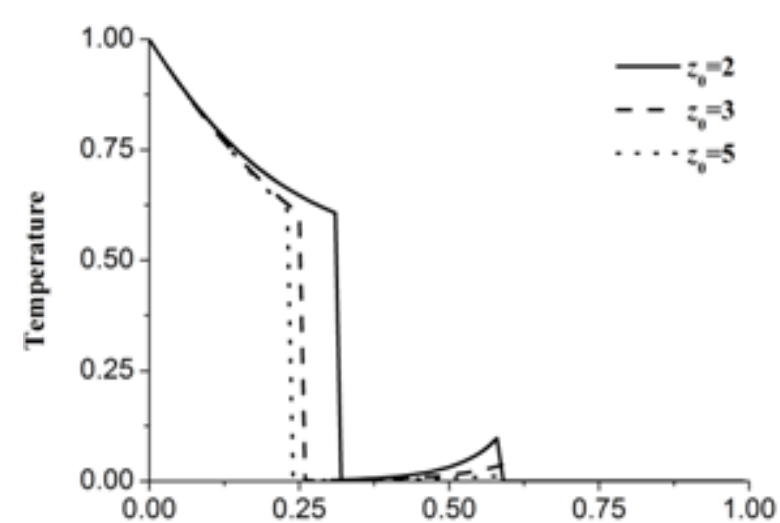

Fig.11. Distribution of temperature $\theta$ versus $x$ with different power law index $z$

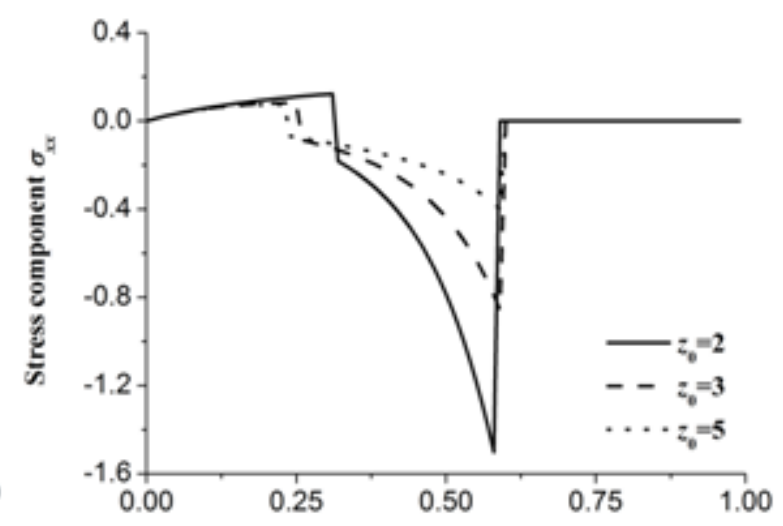

Fig.12. Distribution of stress component $\sigma_{x x}$ versus $x$ with different power law index $z$

\section{Conclusions}

In this paper, an asymptotic approach is proposed to solve the thermoelastic response of FGMs. The governing equations are derived firstly in the context of L-S generalized theory, where the material properties are assumed to vary along the lengthwise direction with a power law. Then the layer method is employed to deal with these nonlinear governing equations, and the linear equations for each layer with the constant material properties are obtained. Finally, the Laplace transform technique and the approximate approach by means of the limit theorem of Laplace transform are used to obtain the asymptotic layer solutions with closed form. This strategy is applied to the solution of a functionally graded thin plate with the boundary subjected to a sudden temperature rise. By the analysis of thermoelastic response, we can have the following results:

1) These asymptotic layer solutions can approach to the real continuous solutions by taking the layer thickness $\Delta x \rightarrow 0$. The explicit expressions of propagation velocities for thermoelastic wave and thermal wave, which are induced by transient thermal shock, can be obtained from these asymptotic solutions, and is convenient to reveal the effect of each characteristic parameter on propagations of two waves.

2) The jumps included in the distributions of temperature and stresses can be accurately captured. The explicit expressions of peak stresses are also obtained, which reveal the function relations between the peak stress and each characteristic parameter and is very important to evaluate the thermo-mechanical properties of FGMs. 
3) Both the thermal relaxation time $\tau_{0}$ and the power law index $z_{0}$ have significant effect on the propagations of each wave and the distributions of displacement, temperature and stresses. It is very necessary to consider the synthetic effect of each characteristic parameter when analyzing the thermoelastic response of FGMs.

\section{Acknowledgement}

This study is sponsored by the National Science Foundation of China (Grant Nos. 51206062, 11102073), the Jiangsu Overseas Research \& Training Program for University Prominent Young \& Middle-aged Teachers and Presidents, and the Priority Academic Program Development of Jiangsu Higher Education Institutions.

\section{References}

[1] Wetherhold RC, Wang SS. The use of functionally graded materials to eliminate or control thermal deformation. Compos Sci Tech 1996; 56: 1099-1104.

[2] Obata Y, Noda N, Tsuji T. Steady thermal stresses in a functionally gradient material plate. Trans of JSME Ser A 1992; 58: 1689-1695.

[3] Obata Y, Noda N. Steady thermal stresses in a hollow circular cylinder and a hollow sphere of a functionally graded material. J Therm Stresses 1994; 17: 471-487.

[4] Tanaka K, Tanaka Y, Watanabe H, Poterasu VF, Sugano Y. An improved solution to thermoelastic material design in functionally gradient materials: Scheme to reduce thermal stresses. Comput Methods Appl Mech Engrg 1993; 109: 377-389.

[5] Bakhshi M, Bagri A, Eslami MR. Coupled thermoelasticity of functionally graded disk. Mech Adv Mater Struct 2006; 13: 219-225.

[6] Shao ZS, Wang TJ, Ang KK. Transient thermo-mechanical analysis of functionally graded hollow circular cylinders. J Therm Stresses 2007; 30: 81-104.

[7] Biot MA. Thermoelasticity and irreversible thermodynamic. J Appl Phys 1956; 27: 240-253.

[8] Lord HW, Shulman Y. A generalized dynamic theory of thermoelasticity. J Mech Phys Solids 1967; 15: 299-309. 
[9] Green AE, Lindsay KA. Thermoelasticity. J Elast 1972; 2: 1-7.

[10]Green AE, Naghdi PM. Thermoelasticity without energy dissipation. J Elast 1993; 31: 189-208.

[11] Youssef HM. Theory of fractionally order generalized thermoelasticity. J Heat Trans 2010; 132: 061301-7.

[12]Povstenko YZ. Fractional cattaneo-type equations and generalized thermoelasticity. J Therm Stresses 2011; 34: 97-114.

[13] Sherief HH, El-Sayed AMA, Abd El-latief AM. Fractional order theory of thermoelasticity. Int J Solids Struct 2010; 47: 269-275.

[14]Bagri A, Eslami MR. Generalized coupled thermoelasticity of functionally graded annular disk considering the Lord-Shulman theory. Compos Struct 2008; 83: 168-179.

[15]Darabseh T, Yilmaz N, Bataineh M. Transient thermoelasticity analysis of functionally graded thick hollow cylinder based on Green-Lindsay model. Int J Mech Mater Des 2012; 8: $247-255$

[16]Ghosh MK, Kanoria M. Analysis of thermoelastic response in a functionally graded spherically isotropic hollow sphere based on Green-Lindsay theory. Acta Mech 2009; 207: $51-67$.

[17]Mallik SH, Kanoria M. Generalized thermoelastic functionally graded solid with a periodically varying heat source. Int J Solids Struct 2007; 44: 7633-7645.

[18]Babaei MH, Chen ZT. Transient thermopiezoelectric response of a one-dimensional functionally graded piezoelectric medium to a moving heat source. Arch Appl Mech 2010; 80: 803-813.

[19] Kanoria M, Ghosh MK. Study of dynamic response in a functionally graded spherically isotropic hollow sphere with temperature dependent elastic parameters. J Therm Stresses 2010; 33: 459-484.

[20] Abbas IA. Nonlinear transient thermal stress analysis of thick-walled FGM cylinder with temperature-dependent material properties. Meccanica 2014; 49: 1687-1708.

[21] Tanigawa Y. Some basic thermoelastic problems for nonhomogeneous structural materials. Appl Mech Rev 1995; 48: 287-300

[22] Tanigawa Y. Theoretical approach of optimum design for a plate of functionally gradient 
materials under thermal loading thermal shock and fatigue behavior of advanced ceramics. NATO ASI Ser E 1992; 241: 171-180

[23]El-Maghraby NM, Youssef HM. State space approach to generalized thermoelastic problem with thermomechanical shock. Appl Math Comput 2004; 156: 577-586.

[24]Abbas IA. Eigenvalue approach in a three-dimensional generalized thermoelastic interactions with temperature-dependent material properties. Comput Math Appl 2014; 68: 2036-2056.

[25]El-Karamany AS, Ezzat MA. Thermal shock problem in generalized thermoviscoelasticity under four theories. Int J Eng Sci 2004; 42: 649-671.

[26] Wang YZ, Zhang XB, Song XN. A unified generalized thermoelasticity solution for the transient thermal shock problem. Acta Mech 2012; 223: 735-743.

[27] Tian XG, Shen YP, Chen CQ, He TH. A direct finite element method study of generalized thermoelastic problems. Int J Solids Struct 2006; 43: 2050-2063.

[28]Balla M. Analytical study of the thermal shock problem of a half-space with various thermoelastic models. Acta Mech 1991; 89: 73-92.

[29] Wang YZ, Zhang XB, Liu D. Asymptotic analysis of generalized thermoelasticity for axisymmetric plane strain problem with temperature-dependent material properties. Int $\mathbf{J}$ Appl Mech 2013; 5: 1350023-20. 